\title{
Nanoscale density variations induced by high energy heavy ions in amorphous silicon nitride and silicon dioxide
}

\section{Mota-Santiago, $\mathrm{P}$.}

2018-04-06

Mota-Santiago , P , Vazquez, H, Bierschenk, T , Kremer , F , Nadzri , A, Schauries , D , Djurabekova , F , Nordlund , K , Trautmann , C , Mudie , S , Ridgway , M C \& Kluth , P 2018 , ' Nanoscale density variations induced by high energy heavy ions in amorphous silicon nitride and silicon dioxide ' , Nanotechnology , vol. 29 , no. 14 , 144004 . https://doi.org/10.1088/1361-6528/aaabdb

http://hdl.handle.net/10138/308629

https://doi.org/10.1088/1361-6528/aaabdb

acceptedVersion

Downloaded from Helda, University of Helsinki institutional repository.

This is an electronic reprint of the original article.

This reprint may differ from the original in pagination and typographic detail.

Please cite the original version. 


\title{
Nanoscale density variations induced by high energy heavy ions in amorphous silicon
}

\section{nitride and silicon dioxide.}

\author{
P. Mota-Santiago ${ }^{1}$, H. Vazquez ${ }^{2}$, T. Bierschenk ${ }^{1}$, F. Kremer ${ }^{3}$, A. Nadzri ${ }^{1}$, D. Schauries ${ }^{1}$, F. Djurabekova ${ }^{2}$, \\ K. Nordlund ${ }^{2}$, C. Trautmann ${ }^{4,5}$, S. Mudie ${ }^{6}$, M. C. Ridgway ${ }^{1}$ and P. Kluth ${ }^{1}$
}

${ }^{1}$ Department of Electronic Materials Engineering, Research School of Physics and Engineering, Australian National University, Canberra ACT 2601, Australia

${ }^{2}$ Helsinki Institute of Physics and Department of Physics, University of Helsinki, PO Box 43, Helsinki, Fl-00014

${ }^{3}$ Centre for Advanced Microscopy, 131 Garran Road, Acton, 2601, Australia

${ }^{4}$ Materials Research Department, GSI Helmholtzzentrum, 64291Darmstadt, Germany

${ }^{5}$ Technische Universität Darmstadt, 64287 Darmstadt, Germany

${ }^{6}$ Australian Synchrotron, 800 Blackburn Road, Clayton VIC 3168, Australia

The cylindrical nanoscale density variations resulting from the interaction of $185 \mathrm{MeV}$ and 2.2 GeV Au ions with $1.0 \mu \mathrm{m}$ thick amorphous $\mathrm{SiN}_{\times}: \mathrm{H}$ and $\mathrm{SiO}_{\times}: \mathrm{H}$ layers is determined using small angle X-ray scattering measurements. The resulting mean density profile resembles an under-dense core surrounded by an over-dense shell with a smooth transition between the two regions, consistent with molecular-dynamics simulations. For amorphous $\operatorname{SiN}_{\mathrm{x}}: \mathrm{H}$, the density variations show a radius of $4.2 \mathrm{~nm}$ with a relative density change larger than three times the value determined for amorphous $\mathrm{SiO}_{x}: \mathrm{H}$, with a radius of $5.5 \mathrm{~nm}$. Complementary infrared spectroscopy measurements exhibit a damage cross-section comparable to the core dimensions. The morphology of the density variations results from freezing in the local viscous flow arising from the non-uniform temperature profile in the radial direction of the ion path. The concomitant drop in viscosity mediated by the thermal conductivity appears to be the main driving force rather than the presence of a density anomaly. 


\section{INTRODUCTION}

Ion irradiation has been extensively studied for the fabrication and modification of materials and nanostructures and is an industrial method in mainstream microelectronics industries. Amongst the many applications, an example is the use of swift heavy ions (SHIs) for the formation of optical waveguides in insulating materials, such as amorphous $\mathrm{SiO}_{2}$ and $\mathrm{Si}_{3} \mathrm{~N}_{4}$, as detrimental effects are significantly reduced in comparison to low-energy ion implantation [1]. At high ion energies, processes are predominantly based on the interaction of the ions with the electrons in the material as the interaction cross section for nuclear collisions dramatically decreases with increasing ion energy. In particular in amorphous insulators, there is still a lack of understanding of the structural transformations caused by intense electronic energy loss, which needs to be addressed for an efficient application of SHIs for the development of advanced materials.

Cylindrical nanoscale density variations are the residual response of insulator materials to the manystage interaction with energetic ions [2] [3]. When swift heavy ions penetrate a solid, intense electronic excitations are produced in narrow regions along the ion paths. The energy exchanged between the ions and the electrons in the material generates energetic electron cascades moving radially outwards, responsible for the temperature profile, interacting with the atoms in the lattice via electron-phonon coupling. The energy deposited in the lattice leads to a rapid local increase in temperature, a so called "thermal spike" that can exceed the melting temperature of the material in a narrow region around the ion trajectory and thus yield the formation of a molten region [4] [5]. The following rapid resolidification can leave a trail of permanent damage that is referred to as an "ion track".

In amorphous materials, the lack of contrast has limited the study and characterization to indirect techniques such as infrared spectroscopy or ion track etching [6]. In the first case, a damage crosssection can be estimated under the assumption that the radiation damage is homogenously distributed along a cylindrical region defined by the ion passage, while the second erases the initial morphology or residual material response. Recently, the ion track morphology of thermally grown $\mathrm{SiO}_{2}$ was determined by synchrotron based techniques, where an under-dense core surrounded by an overdense shell was reported, yet the absolute density change was not determined [2]. Furthermore, the question remained open if the core-shell structure can be ascribed to the density anomaly of $\mathrm{SiO}_{2}$ above $1800 \mathrm{~K}$ [7].

The competition between heat and mass transport, together with the rapid quenching of the thermal spike have a major impact on the ion track morphology [8]. The temperature profile in the thermal spike leads to non-uniform thermal stresses and internal mass transfer [4] [5]. In amorphous materials, 
such as metallic glasses, if the temperature in the ion track region surpasses a critical value, the material experiences a local abrupt decrease in the viscosity [9]. The compressive strains are redistributed providing a purely hydrostatic stress. During the cooling period, the track relaxation kinetics depends strongly on the viscosity value of the material. As the process occurs from the ion track boundary towards the center progressively, it is possible for the generated strains to undergo a reversal process. If the ion track cooling down process is quicker than the density oscillation time, the density fluctuations are only partially restored and a spatial density profile with an under-dense core surrounded by an over-dense shell is expected [9].

Silicon nitride possess a higher dielectric constant, density, melting point and thermal conductivity while exhibiting a band gap of nearly half the value than amorphous $\mathrm{SiO}_{2}\left(\mathrm{a}-\mathrm{SiO}_{2}\right)$ therefore, a suitable material to study the underlying mechanisms involved in the ion track formation process in amorphous materials. For amorphous silicon nitride $\left(\mathrm{a}-\mathrm{Si}_{3} \mathrm{~N}_{4}\right)$, the morphology of ion tracks has been characterized previously only in very thin $(30 \mathrm{~nm})$ layers grown by low pressure chemical vapor deposition (LPCVD) [10]. Here, a similar structure comprised of an under-dense core and an overdense shell was observed by HAADF-STEM. In this case, the nature of the core and shell regions were ascribed to the quenching of the boiling and melting phase, respectively [11], also known as the twothreshold model which is a modified version of the two-temperature model where the core region is defined by the region where boiling temperature is reached. From their experimental results, the determined ion track radius is matched with the numerical calculation of the molten region by choosing the appropriate value of the electronic mean free path, thus the boundary of the core region defines the boiling energy, which for $\mathrm{a}-\mathrm{Si}_{3} \mathrm{~N}_{4}$ was determined to be $2.5 \mathrm{eV} / \mathrm{atom}$.

In this work, we determined the absolute density variation profile generated after irradiation with 185 $\mathrm{MeV}$ and $2.2 \mathrm{GeV}$ Au ions of $1.0 \mu \mathrm{m}$ thick a-SiN : $\mathrm{H}$ and a-SiO $\mathrm{x}: \mathrm{H}$ layers by small angle X-ray scattering (SAXS). Molecular dynamics (MD) simulations were performed for comparison. The experimental and theoretical results agree with a density profile defined by an under-dense core surrounded by an overdense shell with a smooth transition connecting the two regions. We suggest the nature of the over/under-densification process results from the mass and defects redistribution induced by the nonhomogeneous temperature profile caused by the thermal spike. MD simulations reveal a change in the coordination order in the inner region decreasing towards the boundaries of the ion track. A damage cross-section study based on infrared spectroscopy experiments shows good agreement between the coordination-defect distribution calculated by MD simulations and the ion track core region measured by SAXS. 


\section{EXPERIMENTAL}

\section{A. Synthesis and ion irradiation of thin layers}

Layers with $1.0 \mu \mathrm{m}$ thickness of a-SiN $\mathrm{x}: \mathrm{H}$ and a-SiO ${ }_{\mathrm{x}}: \mathrm{H}$ were deposited on aSi (100) wafer by plasma enhanced chemical vapor deposition (PECVD) at $600^{\circ} \mathrm{C}$, with the chemistry of $8: 14: 980: 0$ ( $\mathrm{SiH}_{4}: \mathrm{NH}_{3}: \mathrm{N}_{2}: \mathrm{N}_{2} \mathrm{O}$ in sccm) for a-SiN $\mathrm{N}_{\mathrm{x}}: \mathrm{H}$ and 8:0:270:710 for a-SiO $: \mathrm{H}$ following the deposition conditions presented by Karouta et al. [12], for silicon oxynitrides.

The ion tracks were produced by irradiation with $185 \mathrm{MeV}$ Au ions at the Heavy lon Accelerator Facility (HIAF) at the Australian National University and with $2.2 \mathrm{GeV}$ Au ions at the UNILAC accelerator at GSI in Darmstadt, Germany. Irradiations were performed at room temperature and normal incidence at fluences ranging between $1 \times 10^{11} \mathrm{~cm}^{-2}$ and $5 \times 10^{12} \mathrm{~cm}^{-2}$. The corresponding electronic energy losses $S_{e}$ were calculated with the SRIM2008 code [13], exhibiting an almost constant value across the layer thicknesses $\left(\Delta S_{e} \leq 0.5 \%\right.$ ), supporting the notion of homogeneous ion tracks in the samples. Material properties such as density, thickness and stoichiometry were determined by a combination of Rutherford backscattering spectrometry (RBS), carried out with alpha particles at $2.5 \mathrm{MeV}$ for a-SiN $\mathrm{x}: \mathrm{H}$ (to guarantee no oxygen incorporation or oxidation above the detection threshold during the deposition [14]) and $2 \mathrm{MeV}$ in the case of a-SiO$: \mathrm{H}$, and spectral reflectometry using a SCl Filmtek 400 (with a spectral range between 550 to $1660 \mathrm{~nm}$ ) at different angles. The optical band gap was measured using the Tauc-Lorentz [15] [16] dispersion function. A summary of the measured properties and irradiation conditions is listed in Table 1.

\section{B. Small angle $\mathrm{X}$-ray scattering}

The ion track morphology was studied using synchrotron based transmission SAXS. SAXS possess high sensitivity to small electron density changes; such changes exhibit strong contrast and can be correlated to the morphology of the ion tracks [2] [3] [17]. The measurements were performed at the SAXS/WAXS beamline of the Australian Synchrotron with an X-ray energy of $11 \mathrm{keV}$ and a camera length of $960 \mathrm{~mm}$. Samples were prepared by thinning the Si substrate down to $100 \mu \mathrm{m}$ thickness by mechanical polishing to reduce parasitic scattering from the substrate yet provide enough support for the layers. Data acquisition was carried out with the X-ray beam aligned to the ion track axis and then tilted $10^{\circ}$ off the normal. The absolute calibration of the scattering cross-section was carried out by performing measurements of glassy carbon with $1 \mathrm{~mm}$ thickness as a reference [18]. Furthermore, air and glassy carbon measurements at three different exposure times were recorded ( 1,2 and 5 seconds) to account for the linearly dependency of the photon count with exposure time. When the ion tracks are perfectly aligned with respect to the $\mathrm{X}$-ray beam it is possible to observe the azimuthal symmetry present [Figs. 1 (a) and (b)]. When tilted by $10^{\circ}$ an anisotropic scattering pattern is observed due to 
the high aspect ratio of the ion tracks [Figs. 1 (c) and (d)]. The studied scattering intensities were extracted by a selective mask of the streaks shown in the scattering pattern followed by a background subtraction defined by a mask outlined in an angular sector perpendicular to the streaks. At fluences below $5 \times 10^{11} \mathrm{~cm}^{-2}$, ion track overlap is rather negligible as discussed in previous results and confirmed by a stochastic overlap model [19]. The amorphous nature of our samples supports the assumption of an azimuthally symmetry present. While we measure $\sim 10^{7}$ ion tracks, as they are parallel and almost identical (because of the well-defined ion energy and direction), the resulting analysis reveals information about the individual ion track structure averaging out fluctuations on atomic scales [20].

\section{Molecular Dynamics simulations}

We use the two-temperature molecular dynamics model (2T-MD) [21] to analyze ion tracks produced in a- $\mathrm{Si}_{3} \mathrm{~N}_{4}$ and $\mathrm{a}-\mathrm{SiO}_{2}$ after ion irradiation with $185 \mathrm{Au} \mathrm{MeV}$ ions. In this procedure, the energy deposited to the atoms via electronic excitations is obtained by solving the equations of the twotemperature model [22] up to $30 \mathrm{fs}$. The electron energy distribution is calculated using the Waligorski formula for electrons emitted perpendicularly to the ion trajectory [23]. The free electron gas approximation was used to estimate the electronic thermal conductivity and heat capacity for temperatures higher than the values corresponding to the optical band gap $T_{\mathrm{g}}$, as in Dufour et al. [24]. For a- $\mathrm{SiO}_{2}$ we considered the electron-phonon coupling to be $\mathrm{g}=1.25 \times 10^{13} \mathrm{~W} / \mathrm{cm}^{3} \mathrm{~K}$ [25], while for a$\mathrm{Si}_{3} \mathrm{~N}_{4}$ it was estimated from the expression $g=k_{e} / \lambda^{2}$ for the electron-phonon mean-free path values $\lambda=4.3 \mathrm{~nm}$ (estimated from the band gap according to Toulemonde et al. [26]) and $\lambda=3 \mathrm{~nm}$ (the same value as a-SiO${ }_{2}$ ). For the lattice physical properties of a-Si $\mathrm{N}_{4}$, we used the lattice thermal conductivity $\mathrm{K}_{\mathrm{l}}=6 \mathrm{~W} / \mathrm{m} \cdot \mathrm{K}$ [27] [28] [29] and the heat capacity from Ben-Hai et al. [30]. For a-SiO ${ }_{2}$ the lattice parameters are the same as in Dufour et al. [24]. The de Mota MD potential [31] was applied for our simulations for a-Sij $\mathrm{N}_{4}$, and the Watanabe-Samela [32] [33] many-body potential for a-SiO Munetoh [34] potential was also used to confirm the absence of artifacts due to the potential selected and showed agreement with the other potentials. Nonetheless, it is well known that all three potentials fail to reproduce the melting point of the studied materials sufficiently close to the experiment [35]. We overcame this problem by scaling the deposited energy by the factor obtained as the ratio of the MD melting temperature and the experimental one. We simulated the development of the ion track for 100 ps applying the Berendsen boundary temperature control [36].

The a-SiO2 cell was created using the Wooten-Winer-Weaire (WWW) method, and the a-Si3N4 by heating a random stoichiometric mixture of atoms and then cooling it slowly such that the atoms relax towards their local minima. For both methods we followed the procedure described in detail in [37]. The a-SiO ${ }_{2}$ structure produced with the WWW method shows small amount of initial defects $(<1 \%)$. 
The a-Si ${ }_{3} \mathrm{~N}_{4}$ structure produced with the heat and cool method contained even in the initial state (before irradiation) rather significant amount of coordination defects, which is a known problem of Tersoff-type potentials [38]. The dimensions of the cells employed were $263 \AA \times 258 \AA \times 38 \AA$ for a$\mathrm{Si}_{3} \mathrm{~N}_{4}$ and $241 \AA \times 227 \AA \times 46 \AA$ for a-SiO . To account for statistical uncertainties, the presented simulations were averaged over 5 routines for each material.

\section{Infrared spectroscopy}

The structural bond configurations of a-SiN $\mathrm{N}_{0.95}: \mathrm{H}$ and a- $\mathrm{SiO}_{1.85}: \mathrm{H}$ are infrared active, permitting the establishment of the corresponding nature and density. The FTIR measurements were conducted using a Bruker Optics Vertex $80 \mathrm{~V}$ spectrometer with a resolution of $2 \mathrm{~cm}^{-1}$ within the $400-4000 \mathrm{~cm}^{-1}$ range, and with an untreated Si wafer as a reference. The main absorbance signals were normalized to volume and deconvoluted into different Gaussian peaks, to establish their origin and characterize their evolution with fluence following the procedures reported by other authors [39] [40].

\section{RESULTS}

\section{A. SAXS deduced ion track morphology}

The scattering intensities extracted from the narrow streaks are shown in Fig. 2 (a) and (b). The best fit to the data was obtained with a cylindrical core-shell model with a smooth transition between core and shell densities. The model is sketched in Fig. 2 (c), where the scattering amplitude $f(q)$ for the radial density distribution is expressed as:

$$
f(q)=\frac{2 \pi L \rho_{s}}{q}\left[(\rho-1)\left(\left(R_{c}+T_{t}\right) \frac{J_{1}\left(q \cdot\left(R_{c}+T_{t}\right)\right)}{q}+R \frac{J_{1}(q \cdot R)}{q}\right)-\int_{R_{c}}^{R_{c}+T_{t}} \frac{(\rho-1)}{T_{t}} r^{2} J_{0}(q \cdot r) d r\right] .
$$

The length of the ion track $L$ is equal to the layer thickness and $q$ is the radial component of the scattering vector. The factor $\rho$ corresponds to the density change ratio between the core and the shell regions, $\rho_{S}$ is the maximum absolute density change of the shell. $J_{0}$ and $J_{1}$ are the Bessel function of zeroth and first order respectively, $R_{c}$ is the inner radius considered to have a constant density change value $\rho_{c}, T_{t}$ corresponds to the width of the transition region and $S_{t}$ is the shell width. The total track radius corresponds to $R=R_{c}+T_{t}+S_{t}$. We are considering a smooth transition between the core and shell region approximated by a linear function. We considered a normalized Schulz-Zimm distribution for the core dimensions to account for deviations from the proposed model $\left(\sigma_{c}\right)$ while scaling the distribution of the shell region following the method from Kluth et al. [2].

The numerical fits with the proposed scattering amplitude shown in Fig. 2 (a) and (b), describe the observed measurements with high accuracy, for the data resolved in the extended q-range, in comparison with previous models where a sharp transition between the two regions was considered 
[2]. Therefore, we assign the nature of the weak secondary oscillations to the scattering originated in the transition region. The fitted intensities are shown in Figure 3 (a) for $\mathrm{SiN}_{0.95}: \mathrm{H}$ and $\mathrm{SiO}_{1.85}: \mathrm{H}$ with both irradiation energies and a fluence of $1 \times 10^{11} \mathrm{~cm}^{-2}$. The total track radius $R$ for $185 \mathrm{MeV} \mathrm{Au}$ ions extracted from the theoretical fits amounts to $4.2 \pm 0.1 \mathrm{~nm}$ for $\mathrm{SiN}_{0.95}: \mathrm{H}$, and $5.5 \pm 0.1 \mathrm{~nm}$ for $\mathrm{SiO}_{1.85}: \mathrm{H}$; while for $2.2 \mathrm{GeV}$ Au ions, the determined total track radius was $4.1 \pm 0.1 \mathrm{~nm}$ and $5.3 \pm 0.1 \mathrm{~nm}$ for $\mathrm{SiN}_{0.95}: \mathrm{H}$ and $\mathrm{SiO}_{1.85}: \mathrm{H}$, respectively. The value for the total ion track radius determined for $\mathrm{SiO}_{1.85}: \mathrm{H}$ agrees with previous experiments carried out for thermally grown a-SiO ${ }_{2}$ [2] ( $\left.R=5.4 \pm 0.1 \mathrm{~nm}\right)$. The slightly smaller radius for $2.2 \mathrm{GeV}$ compared to $185 \mathrm{MeV}$ Au ions, despite the higher electronic energy loss (23.9 and $20.7 \mathrm{keV} / \mathrm{nm}$ respectively for a-SiN $\mathrm{N}_{0.95}: \mathrm{H}$, while 19.5 and $16.3 \mathrm{keV} / \mathrm{nm}$ for a-SiO $\left.{ }_{1.85}: \mathrm{H}\right)$, can be ascribed to the velocity effect [41]. Despite the higher energy deposition during the ion irradiation, $\mathrm{SiN}_{0.95}: \mathrm{H}$ exhibits a significantly smaller track radius than $\mathrm{SiO}_{1.85}: \mathrm{H}$ in spite of the higher stopping power (20.7 and $16.3 \mathrm{keV} / \mathrm{nm}$, respectively). This behavior can be the result of the higher melting point $\left(\sim 1900^{\circ} \mathrm{C}\right.$ and $\sim 1700^{\circ} \mathrm{C}$, respectively) but we attribute it mainly to the lower value of the optical band gap, which can be as low as $3 \mathrm{eV}$ for nearly stoichiometric silicon nitride [40] and around $5 \mathrm{eV}$ for silicon dioxide [42]. This results in a higher electron-phonon mean-free path leading to a comparatively lower value for the electron-phonon coupling [43]. Information on the ion track morphology is summarized in Table 1.

The absolute density change can be obtained from the absolute scattering intensity by considering $\lim _{q \rightarrow 0} I(q)$. The corresponding absolute SAXS intensity can be re-written as:

$$
I(q=0)=N V^{2} \rho_{s}^{2}\left\{(\rho-1)\left(\frac{R_{c}+T_{t}}{R}\right)+1-\frac{1}{6}(\rho-1)\left[\left(\frac{R_{c}+T_{t}}{R}\right)^{2}+\left(\frac{R_{c}}{R}\right)^{2}+\left(\frac{R_{c}\left(R_{c}+T_{t}\right)}{R^{2}}\right)\right]\right\}^{2} .
$$

The absolute density change is calculated following the existing relation between the $\mathrm{X}$-ray scattering length density (SLD) $\beta$ and the local density variations: $\rho / \rho_{\text {bulk }}=\beta / \beta_{\text {bulk }}$. The calculated absolute density change for $\mathrm{SiN}_{0.95} \mathrm{H}$ was then determined to be $-8.5 \pm 0.3 \% / 2.1 \pm 0.3 \%$ for the core/shell configuration, while $-1.2 \pm 0.1 \% / 0.25 \pm 0.1 \%$ in the case of $\mathrm{SiO}_{1.85}: \mathrm{H}$ when irradiated with $185 \mathrm{MeV} \mathrm{Au}$ ions. A larger density change was determined for $\mathrm{SiN}_{0.95}: \mathrm{H}$ compared to $\mathrm{SiO}_{1.85}: \mathrm{H}$, consistent with the higher scattering intensity present [Fig. 2 (a) and (b)] while exhibiting a smaller ion track radius. The density profiles showing the absolute density change in \% calculated from this equation are shown in Figure 2 (d). The ion track morphology corresponds to a constant under-dense region surrounded by a thin transition region and an over dense shell. The determination of the configuration is supported by the negative values fitted for $\rho$, the minus sign implies an over/under or under/over dense configuration.

\section{B. Molecular dynamics}


Figures 4 (a) and (b) show the relative radial density change distribution calculated by MD simulations across the ion track region $30 \mathrm{ps}$ after the passage of a single Au ion with an energy of $185 \mathrm{MeV}$ in amorphous $\mathrm{Si}_{3} \mathrm{~N}_{4}$ and $\mathrm{SiO}_{2}$. The simulations illustrate the formation of ion tracks with an under-dense core surrounded by an over-dense shell in both cases.

In the 2T-MD formalism, the radial energy distribution is determined by solving two coupled heat transport equations, one for the electronic subsystem and a second one for the atomic lattice. The coupling factor between the electronic and the lattice temperatures, known as the electron-phonon coupling, is commonly determined through the relation $g=k_{e} / \lambda^{2}$, here $k_{e}$ corresponds to the thermal conductivity of the electronic subsystem and $\lambda$ is defined as the electron-phonon mean free path [26]. The first can be approximated by the free electron model while the latter is regularly left as a free parameter, defined by matching the region surpassing the boiling threshold with the experimental value of the ion track radius [11]. On the other hand, the value of $\lambda$ can also be estimated following the empirical rule for amorphizable insulators, between the value of the optical band gap and the electron-phonon mean free path [26]. For a- $\mathrm{Si}_{3} \mathrm{~N}_{4}$, the value for $\lambda$ has been previously reported following the first approximation to be $\lambda=3 \mathrm{~nm}$ [10]. For this value, a total radius of $5.7 \mathrm{~nm}$ with an underdense core of $1.9 \mathrm{~nm}$ was simulated. Under the second approximation, we determined the value for the electron mean-free-path to be $4.3 \mathrm{~nm}$ after resolve experimentally the optical band gap to be $4.9 \pm 0.1 \mathrm{eV}$; the under-dense region of the ion track exhibited then a radius of $1.7 \mathrm{~nm}$ with a total radius of $4.5 \mathrm{~nm}$. The experimental density change profiles were added in Figure 4 (a) showing better agreement with the MD simulations for the ion track radius with the value $\lambda=4.3 \mathrm{~nm}$. The latter suggests a lower electron-phonon coupling value for $a-\mathrm{Si}_{3} \mathrm{~N}_{4}$, leading to the decrease of the energy transferred to the lattice subsystem limiting the melted volume during the ion track formation process. In contrast with a-Sij $\mathrm{N}_{4}, \mathrm{a}-\mathrm{SiO}_{2}$ has been extensively studied under this formalism and a value of $\mathrm{g}=1.25 \times 10^{13} \mathrm{~W} / \mathrm{cm}^{3} \mathrm{~K}$ has been determined, and adopted in our model. We explored the density change profile for the Watanabe-Samela many body interatomic potential, which shows good agreement with the experimental under-dense core $(3.0 \mathrm{~nm})$ and the total ion track radius $(5.9 \mathrm{~nm})$ but exhibits larger density change values (Figure $3(b)$ ). Such behavior can be the result of the frozenin shock waves obtained after 100 ps simulation, however, the material relaxation may in reality proceed for much longer periods. Also, the MD simulations timescale is of the order of hundreds of picoseconds, much smaller than the timescale of atomic diffusion. Thus, MD simulations do not include the diffusion of atoms from the under-dense core towards the over-dense shell and vice-versa and hence the simulated density variations should be consider as an estimate when compared to experiments. 
From the 2T-MD modelling, we also extracted the change in the content of coordination defects for $\mathrm{Si}$ after the passage of an energetic ion for both systems. The numerical model predicts several atoms being displaced outwards, changing environment and ending trapped in their corresponding new position, because of the density waves propagating through both materials. By analyzing the Si environment at different radial distances, before and after the passage of the swift heavy-ion, we determined the production of under- and over-coordinated defects. The coordination number, as well as the relative increase of under- and over-coordinated defects after the irradiation with respect to the unirradiated structure are shown in Figure 4 (a) and (b). We prefer to show the data in terms of relative increase of defects instead of absolute numbers, since we aim to analyze the formation of new defects formed after the swift heavy-ion impact. For a- $\mathrm{Si}_{3} \mathrm{~N}_{4}$, we observe a decrease in the coordination number of $\mathrm{Si}$ within $3 \mathrm{~nm}$ from the ion track axis (Figure 4 (a)); additionally, the distribution of under-and over-coordinated defects are in good agreement with the under-dense and over-dense region within the ion track. In the case of $\mathrm{a}-\mathrm{SiO}_{2}$, the main change in the coordination number for $\mathrm{Si}$ is also encompassed within the first $3 \mathrm{~nm}$ of the ion track (Figure 4 (b)), and remains under-coordinated at distances further than the total track radius. The increase of under- and overcoordinated defects in the over-dense shell is considerably smaller than in a-Si ${ }_{3} \mathrm{~N}_{4}$.

\section{Fourier transform infrared spectroscopy}

Figure 6 shows the FTIR spectra for a- $-\mathrm{Si}_{3} \mathrm{~N}_{4}$ and a- $-\mathrm{SiO}_{2}$ and their respective deconvolution into Gaussian contributions of their main absorbance peak before irradiation. Between the $400-4000 \mathrm{~cm}^{-1}$ range [Fig. $5(a)$ and (b)], the characteristic absorption bands are ascribed to the respective vibrational modes of $\mathrm{Si}-\mathrm{N}$ and $\mathrm{Si}-\mathrm{O}$ and those related to the presence of residual $\mathrm{H}$. For a-SiN $\mathrm{N}_{0.95}: \mathrm{H}$, the main infrared absorption peak is centered at $874 \mathrm{~cm}^{-1}$ and presents a shoulder around $1200 \mathrm{~cm}^{-1}$, which can be deconvoluted into four contributions [Fig. 5 (c)]: (i) around $816 \mathrm{~cm}^{-1}$ the Si-N stretching mode in the $\mathrm{N}-\mathrm{Si}_{3}$ configuration is present, (ii) the main contribution is located at $890 \mathrm{~cm}^{-1}$ and is attributed to the Restsrahlen effect or TO mode: due to the forbidden photon propagation in the IR leading to a high reflectivity [32,33], (iii) the $1040 \mathrm{~cm}^{-1}$ band originates from the asymmetric stretching mode of the $\mathrm{Si}-\mathrm{N}$ bond in the $\mathrm{H}-\mathrm{SiN}_{3}$ configuration, and (iv) near $1200 \mathrm{~cm}^{-1}$ we found the contribution of the N$\mathrm{H}$ rocking mode [14] [39] [44]. At $2180 \mathrm{~cm}^{-1}$ different Si-H vibrational modes are present [40], while around $3340 \mathrm{~cm}^{-1}$ the absorption peaks from $\mathrm{N}-\mathrm{H}_{2}$ asymmetric stretching modes are observed due to the $\mathrm{H}$ content of around 10 atomic percent [12] [14] [39] [44].

The infrared spectrum of a-SiO ${ }_{1.85}: \mathrm{H}$ present two absorption peaks in the region $750-1400 \mathrm{~cm}^{-1}$. The first, centered at $815 \mathrm{~cm}^{-1}$, corresponds to the Si-O-Si bending mode [42] [45] [46]. In the region 900$1400 \mathrm{~cm}^{-1}$ the main absorbance signal centered at $1050 \mathrm{~cm}^{-1}$ encompasses the Si-O-Si stretching and Si-O symmetrical modes, while exhibiting a shoulder at $1200 \mathrm{~cm}^{-1}$ due to the stretching modes 
associated to the $\mathrm{Si}\left(\mathrm{O}_{4}\right)$ configuration [Fig. 5 (d)]. Very weak absorption peaks are located around 2300 $\mathrm{cm}^{-1}$ and $3500 \mathrm{~cm}^{-1}$ corresponding to $\mathrm{Si}-\mathrm{H}$ and SiO-H. The former confirms a very low concentration of H, below 3\% [Fig. 5 (a)].

After irradiation, we observed a decrease in the absorbance of all our samples, which can be correlated to the radiation damage from the swift heavy ion irradiation process (Figure $6(a)$ and (b)). Contrary to previous studies involving IR spectroscopy [47] [48], no significant shift towards higher wavenumbers is observed below a fluence of $5 \times 10^{12} \mathrm{~cm}^{-2}$, where overlapping effects become relevant. A non-uniform decrease of the main absorbance band is observed as a function of the irradiation fluence in both materials. The combined behavior corresponding to $\mathrm{Si}-\mathrm{H}$ and $\mathrm{N}-\mathrm{H}$ bands shows no obvious trend. For example, at $620 \mathrm{~cm}^{-1}$, the Si-H wagging mode is present for both materials [40] [44] yet, no change in intensity is observed while increasing the irradiation fluences. Also, at 2280 and 2250 $\mathrm{cm}^{-1}$ the $\mathrm{Si}-\mathrm{H}$ bending modes of a-Si $\mathrm{Si}_{4}$ and a-SiO $\mathrm{S}_{2}$ show no decrease in absorbance with increasing fluence; instead, a clear increase occurs above $5 \times 10^{12} \mathrm{~cm}^{-2}$ probably a result of the complex behavior of $\mathrm{H}$ and its corresponding interaction with the $\mathrm{Si}$ dangling bonds product of the $\mathrm{Si}-\mathrm{N}$ and $\mathrm{Si}-\mathrm{O}$ rupture due to the irradiation damage [40] [46]. As the density of Si-N or Si-O bonds is related to the stretching modes [24] [44] [45], the decrease in the corresponding integrated absorbance signal is considered a direct result of radiation damage being created by the ion track formation process. For Si-N, the TO mode is indicative of the Si-N density [14], and we considered it to be a direct measurement of the Si$\mathrm{N}$ bond density in our study. In our analysis, we are not considering compaction or swelling effects due to the low fluence values used. To quantify the radiation-induced modification we describe the decrease in the $600-1200 \mathrm{~cm}^{-1}$ integrated absorbance signal by a Poisson distribution [48]:

$$
A_{S i-N / S i-O}(R, F)=C_{S i-N / S i-O}+\left(1-C_{S i-N / S i-O}\right) \cdot \exp \left(-\pi R^{2} F\right),
$$

where the term $A_{S i-N / S i-O}$, corresponds to the integrated absorption bands for both materials at a given fluence and, $C_{S i-N / S i-O}$ is the relative contribution to the absorbance peak, $R$ is the radius of the modified region under the presumption of exhibiting a homogeneous radiation damage density, and $F$ is the irradiation fluence. Figures 6 (c) and (d) show the fitted curves of the corresponding absorbance values for different irradiation fluences normalized to the as-deposited integrated absorbance. Track radii of $1.9 \pm 0.3 \mathrm{~nm}$ and $1.6 \pm 0.2 \mathrm{~nm}$ were determined in the case of $\mathrm{SiN}_{0.95}: \mathrm{H}$ for $185 \mathrm{MeV}$ and $2.2 \mathrm{GeV}$ Au irradiation, respectively, and values of $2.8 \pm 0.4 \mathrm{~nm}$ and $2.2 \pm 0.5 \mathrm{~nm}$ for $\mathrm{SiO}_{1.85}: \mathrm{H}$, respectively. The results show good agreement with the ion track core dimensions deduced from SAXS, supporting the idea of a high damage concentration in the inner region of the ion track.

\section{DISCUSSION}


The complex interaction of an energetic ion with a solid target involves different electronic and atomic processes and occurs at different timescales. The duration of the ion passage is $10^{-18}-10^{-17} \mathrm{~s}$, followed by an electron cascade moving radially outwards between $10^{-15}-10^{-13} \mathrm{~s}$ known as $\delta$-electrons. The excited electrons interact with the target atoms transferring energy from the electronic to the atomic subsystem via the electron-phonon coupling building up a thermal spike, which commonly occurs in insulators between $10^{-13}$ and $10^{-11} \mathrm{~s}$, leading to local melting and finally to the plastic flow of the molten region in the last stage. A key part of the ion track formation process is the thermal spike, which can be described by two coupled heat diffusion equations corresponding to the excited electrons and the atoms in the lattice. We have previously computed the time evolution of the temperature profile based on the i-TS model and the impact of the material properties of the target [49] by following the framework described in [24] with one fundamental difference: we determined experimentally the optical band gap to approximate the electron mean-free-path $(\lambda)$ using the empirical relationship known between them [43] instead of numerically fitting the $\lambda$-value using experimentally determined ion track radii. From the numerical results we observed two fundamental differences in the thermal spike evolution between silicon nitride and silicon dioxide: the difference in the optical band gap is mainly responsible for the variation in the ion track dimensions between the two materials as it leads to an electron-phonon coupling value in a- $\mathrm{Si}_{3} \mathrm{~N}_{4}$ of less than half the value of a-SiO${ }_{2}$ and, the higher thermal conductivity of a-Si $\mathrm{N}_{4}$ (nearly one order of magnitude higher than a-SiO${ }_{2}$ ) reduces the duration of the occurrence of the molten phase in a- $\mathrm{Si}_{3} \mathrm{~N}_{4}$ by one order of magnitude compared to a$\mathrm{SiO}_{2}$.

SAXS experiments offer several advantages over other available techniques to determine with high accuracy the mean ion track morphology in amorphous materials. The density variation profiles determined by SAXS support the time evolution numerically calculated from the $\mathrm{i}$-TS model and the impact of the bulk material properties on the target response. Previous studies on the density evolution within the ion track core region in insulators have shown that in the case of amorphous silicon dioxide, an abrupt decrease in density is achieved around 4 ps followed by a reduction in the density change at 150 ps without a full recovery of the initial density [8]. When the duration of the thermal spike is reduced, it leads to a more efficient energy dissipation and faster freeze-in of the density wave induced by the incident ion. As a consequence, the recovery process is much shorter as for a-Si $\mathrm{N}_{4}$, explaining the high density fluctuations in the core region (of nearly $10 \%$ ) while for silicon dioxide we observe a density change within $2 \%$, in agreement with previous [8] and the present MD simulations. A similar ion track morphology with high density fluctuations in the core region has been observed in very thin layers of low-pressure chemical vapor deposited silicon nitride (thicknesses between $5-100 \mathrm{~nm}$ ) where the variation in density within the ion track region was estimated by 
HAADF-STEM [10]. While the results are in good agreement with our measurements, it is important to highlight that the track length in our experiments is far greater than that $(\sim 1 \mu \mathrm{m})$ and the measurement technique is noninvasive such that we can rule out surface effects and artifacts due to sample preparation and measurement. Our results clearly demonstrate: (i) the track radius in a-Si $\mathrm{N}_{4}$ is smaller than in a-SiO ${ }_{2}$ possibly resulting from the difference in the optical band gap; (ii) the density change is comparably larger in a- $\mathrm{Si}_{3} \mathrm{~N}_{4}$ compared to a-SiO${ }_{2}$ due to a larger thermal conductivity, which leads to shorter relaxation times, i.e. time for the density change to recover; (iii) the ion tracks are continuous with a morphology exhibiting a core-shell density change distribution where the boundary between the under-dense and the over-dense regions is smooth and can be estimated by a linear function satisfactorily rather than an abrupt transition [2], also supported by the 2T-MD density calculations presented.

The 2T-MD density calculations of the radial distribution of the density variations exhibit a morphology of an underdense core surrounded by an over-dense shell with a smooth transition region in between. This occurs because of the over-densification arising from the movement of atoms radially outwards from the center of the ion track, increasing the number of coordination defects in the inner region of the ion track. This answers the open question if the under-dense core/over-dense shell track in $\mathrm{a}_{-} \mathrm{SiO}_{2}$ is a result of the density anomaly above $1800 \mathrm{~K}$ [50] [2] [8].

For amorphous silicon nitride the morphology was ascribed to the high temperature reached in the inner region, surpassing the vaporization point, however no experimental support was provided for this argument [10]. It is in fact questionable if the equilibrium vaporization point is relevant in a spatially enclosed area such as the track area away from the surface where the temperature increase leads to high pressure around the track center. In contrast, our 2T-MD simulations suggested that the results above can be explained by the plastic viscous flow in the framework of the inelastic thermal spike model instead. In this formalism, the swift heavy ion deposits energy in the thin layer via electronic interactions leading to a band gap dependent radial cascade of energetic free electrons [43]. During this process, most of the energy is deposited within a radius of $1 \mathrm{~nm}$ [23]. Part of the energy is diffused by inelastic electron collisions (electronic thermal conductivity) and the rest is transferred to the lattice at later stages through electron-phonon coupling [5]. As discussed by Borodin et al. [9], the determination and evolution of the temperature profile in the ion track play a crucial role. The temperature profile described by the i-TS calculations leads to a non-uniform thermal expansion in the molten track restricted by a cold boundary imposed by the matrix. The generated internal stresses leads to redistribution of matter due to defect diffusion. Although different to amorphous metals, where above a critical temperature $T_{c}$, a glass transition takes place exhibiting a viscosity drop [9], a-SiO ${ }_{2}$ undergoes a transition towards a critical flow state and a drastic decrease in 
viscosity [51] while a-Si ${ }_{3} \mathrm{~N}_{4}$ experiences a transition from ceramics towards a ductile state [52] [53]. At this stage, the density variation, described as a damped density wave, is dependent on the relation between the cooling down period and the oscillation period. In our case, the viscosity cannot be disregarded, therefore the damping constant can exceed the oscillation frequency quenching in a steady-state that partially compensates the thermal strain gradients, similar to the behavior reported in [8]. Thus, the ion track does not fully return to the original state, leaving a trace of redistributed matter.

The information extracted from our 2T-MD results regarding the change in coordination numbers for Si (Fig. 5) shows that the increment in coordination defects inside the ion track region is smoothly decreasing with increasing radial distance from the track origin for both materials. This indicates that the radius of the damage cross-section obtained by our FTIR analysis can be understood as an effective cross-section area where most of the coordination defects are located. Under this approximation, the radius of the effective damage cross-section is comparable with the dimension of the core region for both materials. However, we did not observe a direct relation between the dimension of the damage region define by IR analysis and the total ion track radius, different to what is often observed for the case of amorphizable insulators [11]. This is not surprising as in the latter case a phase change of the material from crystalline to amorphous often defines the track region.

The accurate determination of the morphology of ion tracks in amorphous materials is of crucial relevance in order to understand the corresponding effects on the modification of the material properties. Previous results based on indirect techniques such as electrical conductivity or infrared spectroscopy have been applied to estimate the ion track dimensions in a-Si $\mathrm{S}_{3} \mathrm{~N}_{4}$ and a- $\mathrm{SiO}_{2}$, where concepts developed for crystalline materials were applied. In $\mathrm{a}-\mathrm{SiO}_{2}$, the evolution of the absorbance band located around $1080 \mathrm{~cm}^{-1}$ has been used to study the radiation damage in the local structure induced by SHI [54] [55] [56] [57]. The decrease of the absorbance peak area is considered as direct indication of the bond-breaking after the ion passage and therefore, a direct measurement of the ion track dimensions. However, a study of the $\mathrm{Si}-\mathrm{O}$ bond density evolution as a function of fluence has shown that the modification of the IR signal cannot be uniquely attributed to bond breaking but also to structural deformation and irradiation-induced strain, responsible for the observed red shift and increase in peak width [57]. The red shift of the absorbance band position is indicative of the variation of the Si-O-Si angle, suggesting a decrease in the bond angle as a consequence of the irradiationinduced strain. It is noteworthy that the observed changes in bond angles are mostly reported for fluences above $5 \times 10^{12} \mathrm{~cm}^{-2}$, where superposition becomes relevant [19] and it becomes difficult to correlate the overall irradiation-induced strain to the single ion track morphology. For a- $\mathrm{Si}_{3} \mathrm{~N}_{4}$, only few experiments have been carried out and mainly in very thin layers where the ion track dimensions 
are estimated solely by the decrease of the absorbance peak followed by a shoulder around $900 \mathrm{~cm}^{-1}$ and $1200 \mathrm{~cm}^{-1}$ [48]. The decrease of the absorbance signal was fitted with a single Poisson statistical model to determine the ion-induced damage cross-section for fluences below $5 \times 10^{13} \mathrm{~cm}^{-2}$ while at higher fluences a second damage cross-section was added to account for nuclear interactions, without considering any other aspect in the evolution of the infrared spectra. No observation regarding a red shift of the asymmetric Si-N stretching modes were reported. Our experiments presented in Fig 7, show no apparent shift in the FTIR spectra below $5 \times 10^{12} \mathrm{~cm}^{-2}$, as consequence of the small extent of the radiation damage cross-section. This suggest that the coordination defects, following the radial distribution calculated by Molecular Dynamics, does not lead to an observable effect at low fluences as it is below the detection limit and only becomes important as a collective effect at high fluences where sufficient radiation-induced damage is present.

\section{CONCLUSIONS}

In conclusion, SAXS measurements in combination with 2T-MD simulations and infrared spectroscopy provide not only an accurate picture of the morphology of ion tracks in amorphous silicon nitride and silicon dioxide but also explaine the nature of the underdense core surrounded by an overdense shell morphology with a smooth transition. The morphology is a result of the viscous flow present in the molten region driven by thermal stresses arising from the non-uniform temperature profile. As the decrease of the viscosity in the inner region is not as abrupt as in the case of amorphous metals, the freeze-in process is faster than the oscillation frequency or relaxation period, leading to large density fluctuations. Thus, the larger value of the lattice thermal conductivity of amorphous silicon nitride appears to have a major role in the larger values of the density variations compared to $\mathrm{a}-\mathrm{SiO}_{2}$. The core-shell structure with a smooth transition is then the result of the quenched density wave. 


\section{ACKNOWLEDGMENTS}

P. Mota-Santiago would like to thank the Consejo Nacional de Ciencia y Tencologia (CONACYT). We acknowledge access to NCRIS facilities (ANFF and the Heavy Ion Accelerator Capability) at the Australian National University. This work was performed at the SAXS/WAXS beamline at the Australian Synchrotron. P. Kluth and M. C. Ridgway thank the Australian Research Council for financial support. 
TABLE I. Physical parameters measured from RBS and spectral reflectometry, irradiation parameters including electronic energy loss $S_{e}$ as calculated by SRIM2008 considering $1 \mu \mathrm{m}$ thick layers, and fitting parameters from the SAXS measurements: $R_{c}$ (inner core radius), $T_{t}$ (width of transition region) and $R$ (total ion track radius) for samples irradiated with a fluence of $1 \times 10^{11} \mathrm{~cm}^{-2}$. The term $\sigma_{c}$ corresponds to the polydispersity of the inner core radius, while the ratio $\rho_{c} / \rho_{s}$ represents the ratio between the density change of the inner core region and the density change of the shell section. 
FIG 1. Scattering patterns of tracks formed by $185 \mathrm{MeV} 1 X 10^{11} \mathrm{~cm}^{-2}$ Au ions in a-SiN 0.95 (a) and a-SiO $\mathrm{S}_{1.85}$ (b) aligned and tilted with respect to the $X$-ray beam by $10^{\circ}(\mathrm{c})$ and $(\mathrm{d})$ respectively.

FIG 2. Numerical fit of the integrated SAXS scattering intensities for samples irradiated with $185 \mathrm{MeV}$ (filled markers) and $2.2 \mathrm{GeV}$ (open markers) Au ions with fluences of $1 \times 10^{11} \mathrm{~cm}^{-2}$ for a-SiN 0.95 (a) and a$\mathrm{SiO}_{1.85}$ (b), solid lines represent the numerical fit considering a core-shell with a linear transition model. (c) Diagram of the core-shell model adopted with a linear density transition between core and shell. (d) Corresponding normalized density change profile extracted from the numerical calculations for ion tracks formed in a-SiN 0.95 and a-SiO 1.85 .

FIG 3. Normalized radial density change calculated by molecular dynamics simulations after 30 ps. and their corresponding comparison with the normalized density profile determined from SAXS for: (a) a$\mathrm{Si}_{3} \mathrm{~N}_{4}(\mathrm{MD})$ and $\mathrm{SiN}_{0.95}(\mathrm{SAXS})$, (b) a-SiO 2 (MD) and $\mathrm{SiO}_{1.85}$ (SAXS).

FIG 4. The left axis corresponds to the coordination number of Si (solid lines) for $a-S_{3} \mathrm{~N}_{4}(a)$ and $a-\mathrm{SiO}_{2}$ (b), while the right axis represents the relative increase of under- and over-coordination defects (dashed lines) after the passage of one $185 \mathrm{MeV}$ Au ion, both as a function of the distance from the ion track center.

FIG 5. FTIR absorbance spectra of unirradiated a-SiN $\mathrm{S}_{0.95}: \mathrm{H}(\mathrm{a})$ and $\mathrm{a}-\mathrm{SiO}_{1.85}: \mathrm{H}(\mathrm{b})$ and the corresponding deconvolution (b) and (d) in Gaussian contributions of the principal signal between $600-1200 \mathrm{~cm}^{-1}$.

FIG 6. FTIR spectra for various fluences for the wavenumber range $2000-3600 \mathrm{~cm}^{-1}$. The absorbance was multiplied by $5 x$ for clarity for $\mathrm{SiN}_{0.95}: \mathrm{H}$ (a) and $\mathrm{SiO}_{1.85}: \mathrm{H}(\mathrm{b})$. Absorbance of Si-N (c) and Si-O (d) bands as a function of fluence with the lines representing exponential fits based on the Poisson law described above. 
Table 1

\begin{tabular}{|c|c|c|c|c|c|c|c|c|c|c|}
\hline \multirow[b]{2}{*}{ Sample } & \multicolumn{3}{|c|}{ Physical properties } & \multicolumn{2}{|c|}{ Irradiation conditions } & \multicolumn{5}{|c|}{ Morphology } \\
\hline & $\mathrm{x}$ & $\begin{array}{l}\text { Density } \\
\left(\mathrm{g} / \mathrm{cm}^{3}\right)\end{array}$ & $\begin{array}{c}\mathrm{n} \\
@ 600 \\
\mathrm{~nm}\end{array}$ & $\begin{array}{l}\text { Energy } \\
(\mathrm{MeV})\end{array}$ & $\begin{array}{c}S_{e} \\
(\mathrm{keV} / \mathrm{nm})\end{array}$ & $\begin{array}{c}\mathrm{R}_{\mathrm{c}} \\
(\mathrm{nm})\end{array}$ & $\begin{array}{c}T_{t} \\
(n m)\end{array}$ & $\begin{array}{c}\mathrm{R} \\
(\mathrm{nm})\end{array}$ & $\begin{array}{c}\sigma_{c} \\
(n m)\end{array}$ & $\rho_{c} / \rho_{s}$ \\
\hline \multirow[b]{2}{*}{$\operatorname{SiN}_{x}$} & \multirow[b]{2}{*}{$0.95 \pm 0.03$} & \multirow[b]{2}{*}{$2.72 \pm 0.02$} & \multirow[b]{2}{*}{1.95} & 185 & 20.7 & $1.5 \pm 0.1$ & $1.2 \pm 0.1$ & $4.2 \pm 0.1$ & $0.5 \pm 0.1$ & $-4.02 \pm 0.02$ \\
\hline & & & & 2300 & 23.9 & $1.3 \pm 0.1$ & $1.1 \pm 0.1$ & $4.1 \pm 0.1$ & $0.4 \pm 0.1$ & $-4.51 \pm 0.02$ \\
\hline \multirow{2}{*}{$\mathrm{SiO}_{x}$} & \multirow{2}{*}{$1.85 \pm 0.02$} & \multirow{2}{*}{$2.15 \pm 0.02$} & \multirow{2}{*}{1.48} & 185 & 16.3 & $1.7 \pm 0.1$ & $1.2 \pm 0.1$ & $5.5 \pm 0.1$ & $0.3 \pm 0.1$ & $-4.92 \pm 0.02$ \\
\hline & & & & 2300 & 19.5 & $1.8 \pm 0.1$ & $0.9 \pm 0.1$ & $5.3 \pm 0.1$ & $0.2 \pm 0.1$ & $-5.02 \pm 0.02$ \\
\hline
\end{tabular}


Figure 1

a)

b)

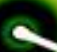

c)

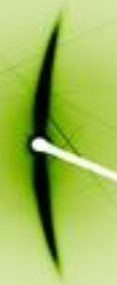

d) 
Figure 2
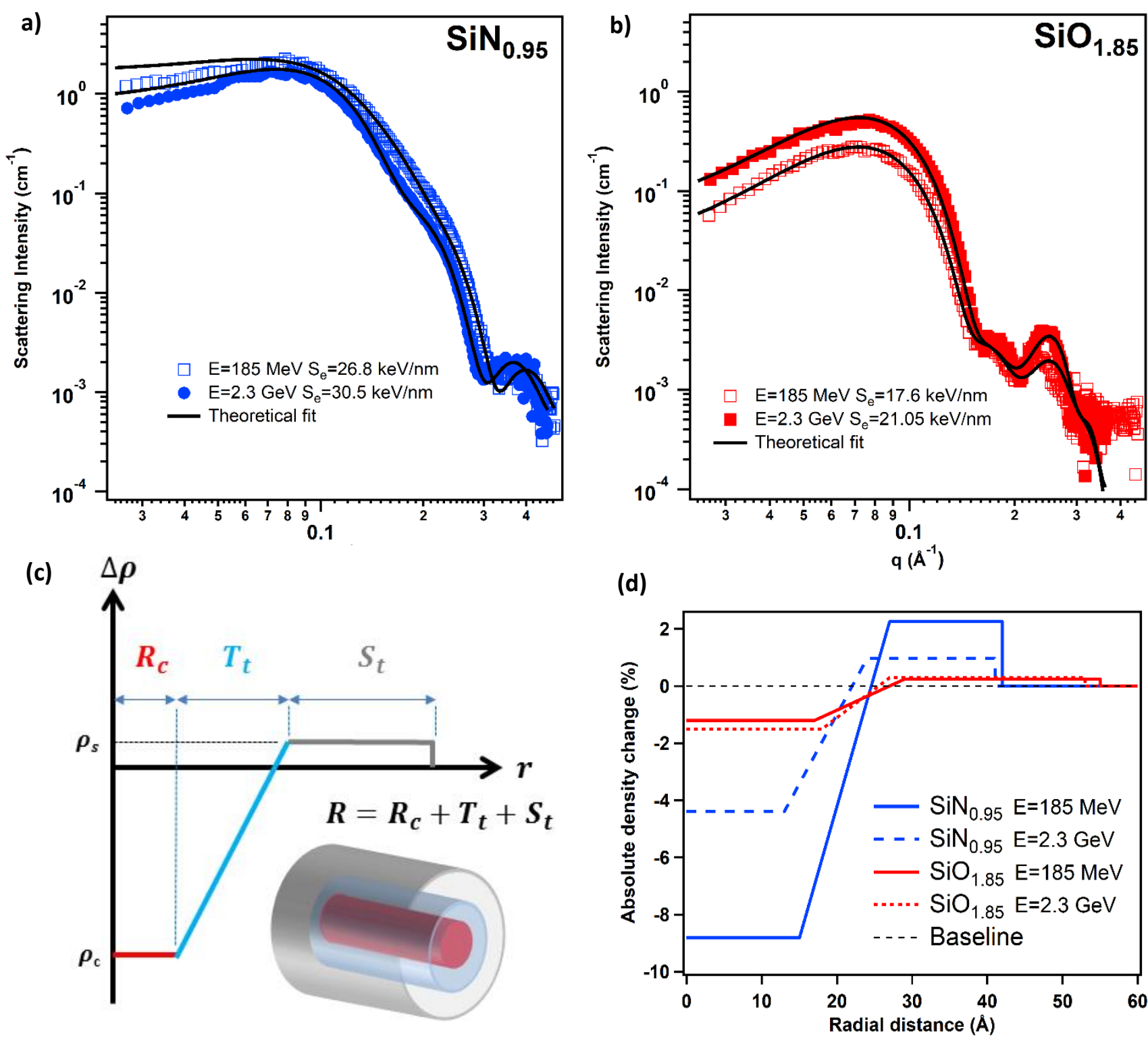
Figure 3

a)

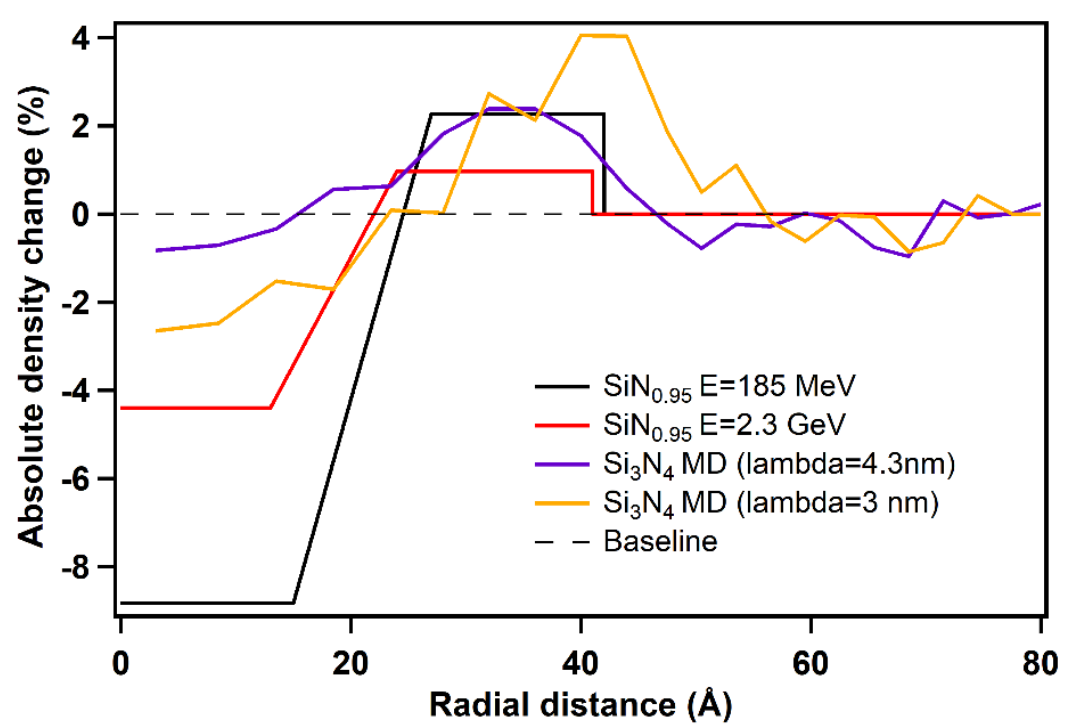

b)

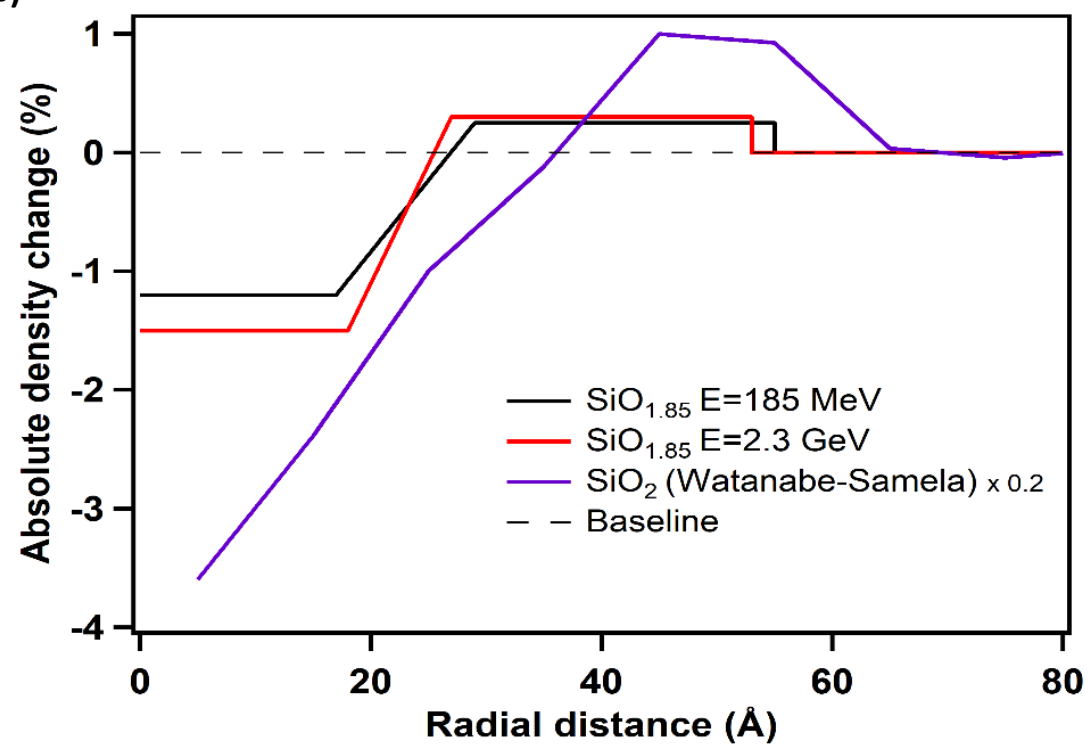


Figure 4
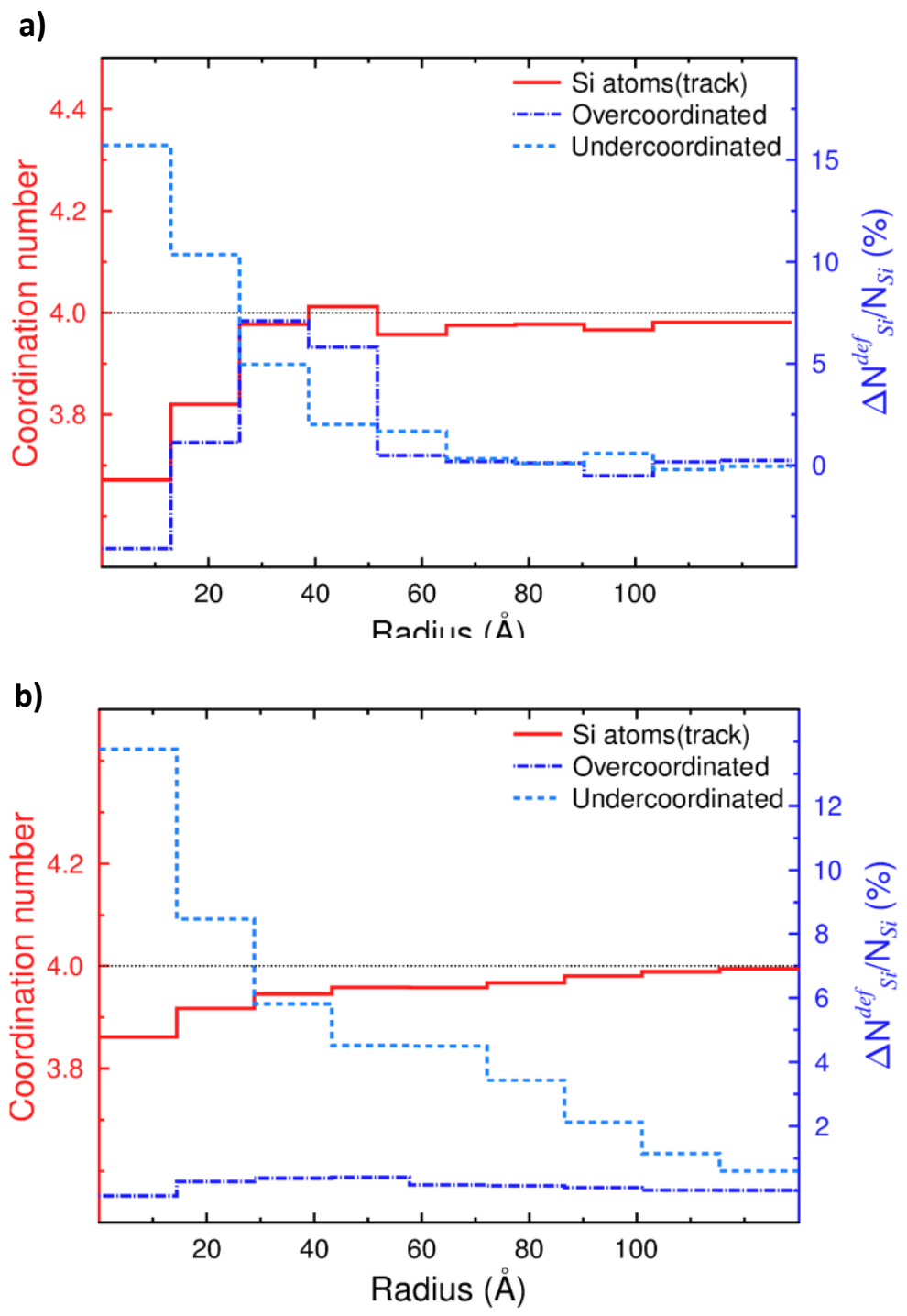
Figure 5
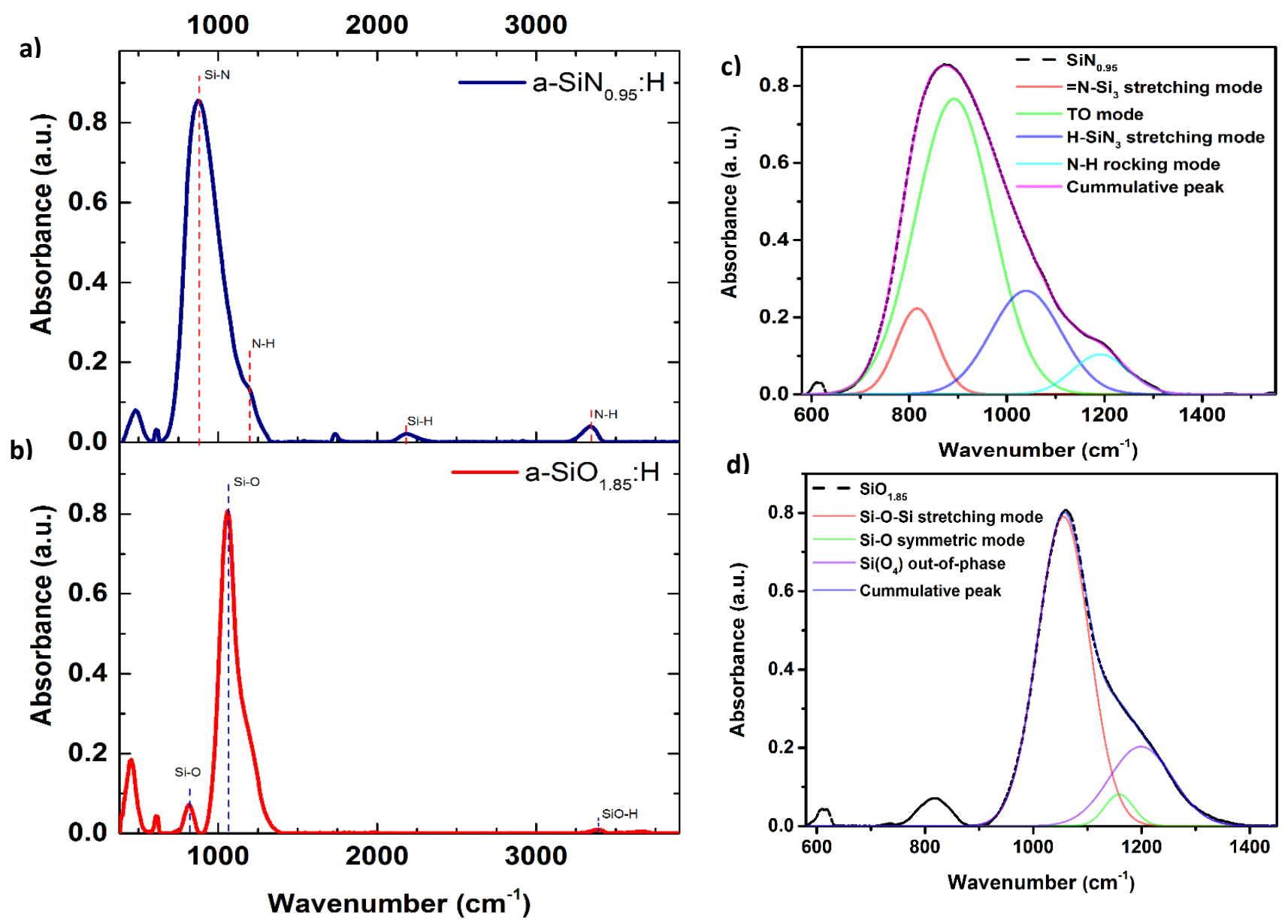
Figure 6
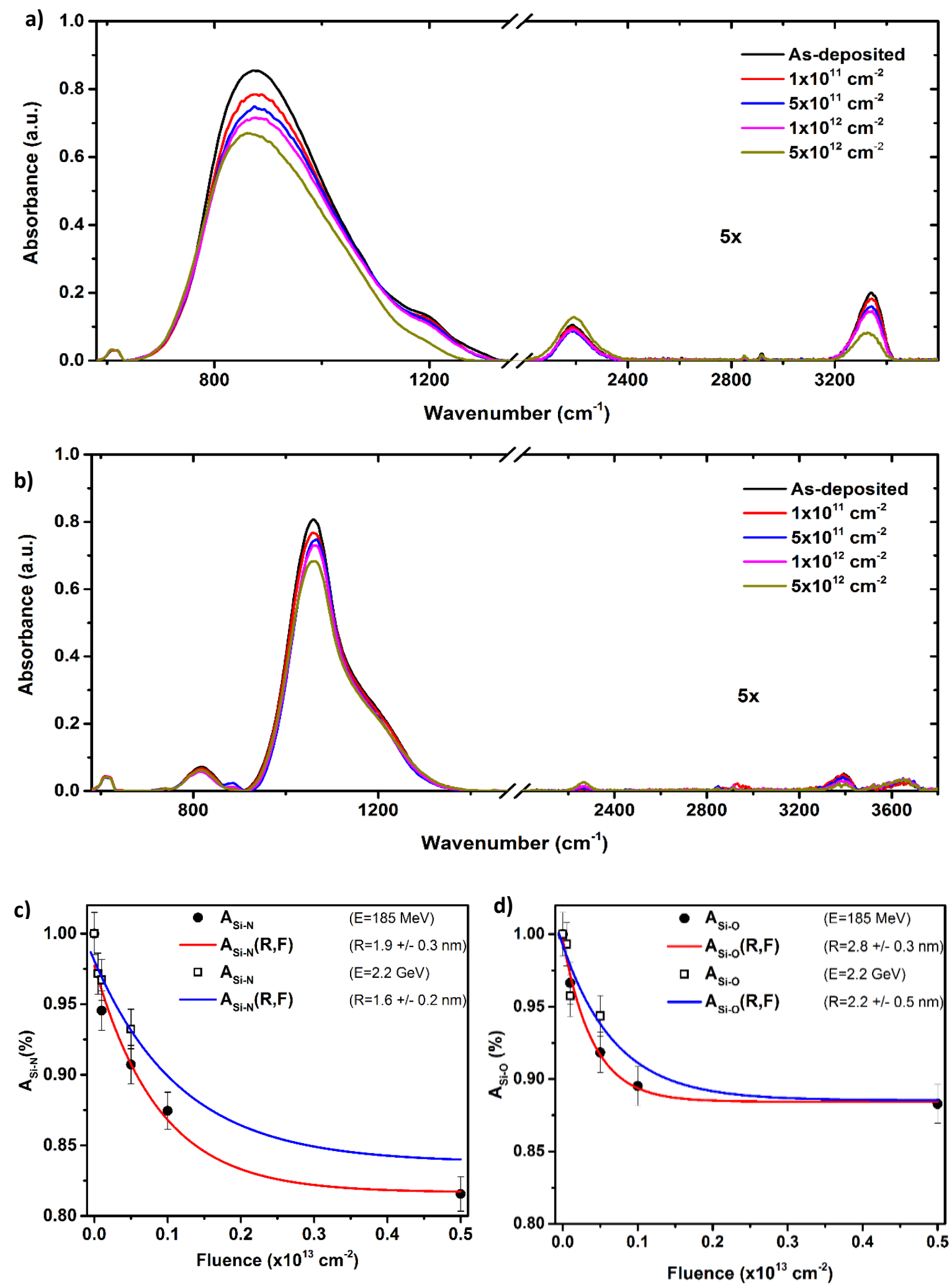


\section{References}

[1] O. Peña-Rodriguez, J. Olivares, M. Carrascosa, A. Garcia-Cabañes, A. Rivera and F. Agullo-Lopez, "Optical Waveguides Fabricated by Ion Implantatio/Irradiation: A review," in Ion Implantation, P. M. Goorsky, Ed., InTech, 2012, pp. 267-314.

[2] P. Kluth, C. S. Schnohr, O. H. Pakarinen, F. Djurabekova, D. J. Sprouster, R. Giulian, M. C. Ridgway, A. P. Byrne, C. Trautmann, D. J. Cookson, K. Nordlund and M. Toulemonde, "Fine Structure in Swift Heavy Ion Tracks in Amprhous SiO2," Phys. Rev. Lett., vol. 101, no. 17, p. 175503, 2008.

[3] T. Bierschenk, R. Giulian, B. Afra, M. D. Rodriguez, D. Schauries, S. Mudie, O. H. Pakarinen, F. Djurabekova, K. Nordlund, O. Osmani, N. Medvedev, B. Rethfeld, M. C. Ridgway and P. Kluth, "Latent ion tracks in amorphous silicon," Phys. Rev. B, vol. 88, no. 17, p. 174111, 2013.

[4] H. Trinkaus and A. I. Ryazanov, "Viscoelastic model for the plastic flow of amorphous solids under energetic ion bombardment," Phys. Rev. Lett., vol. 74, no. 25, pp. 5072-5075, 1995.

[5] A. I. Ryazanov, A. E. Volkov and S. Klaumünzer, "Model of track formation," Phys. Rev. B, vol. 51, no. 18, pp. $12107-12$ 115, 1995.

[6] C. Trautmann, S. Klaumünzer and H. Trinkaus, "Effect of Stress on Track Formation in Amorphous Iron Boron Alloy: Ion Tracks as Elastic Inclusions," Phys. Rev. Lett., vol. 85, no. 17, p. 3648, 2000.

[7] J. E. Shelby, Introduction to Glass Science and Technology, Cambridge: The Royal Society of Chemistry, 1997.

[8] O. H. Pakarinen, F. Djurabekova and K. Nordlund, "Density evolution in formation of swift heavy ion tracks in insulators," Nucl. Instr. Meth. Phys. Res. B, vol. 268, pp. 3163-3166, 2010.

[9] V. A. Borodin, A. E. Volkov and D. N. Korolev, "Viscous flow of amporphous metals in swift heavy ion tracks," Nucl. Instr. Meth. Phys. Res. B, vol. 209, pp. 122-130, 2003.

[10] T. Kitayama, Y. Morita, K. Nakajima, K. Narumi, Y. Saitoh, M. Matsuda, M. Sataka, M. Tsujimoto, S. Isoda, M. Toulemonde and K. Kimura, "Formation of ion tracks in amorphous silicon nitride films with MeV C60," Nucl. Instr. Meth. Phys. Res. B, Vols. 356-357, pp. 22-27, 2015.

[11] M. Toulemonde, A. Benyagoub, C. Trautmann, N. Khalfaoui, M. Boccanfuso, C. Dufour, F. Gourbilleau, J. J. Grob, J. P. Stoquert, J. M. Constantini, F. Haas, E. Jacquet, K.-O. Voss and A. Meftah, "Dense and nanometric electronic excitations induced by swift heavy ions in an ionic CaF2 crystal: Evidence for two thresholds of damage creation," Phys. Rev. B, vol. 852, no. 5-1, p. 054112, 2012.

[12] F. Karouta, K. Vora, J. Tian and C. Jagadish, "Structural, compositional and optical properties of PECVD silicon nitride layers," J. Phys. D, vol. 45, p. 445301 (10 pp), 2012. 
[13] J. F. Ziegler., J. P. Biersack and U. Littmark, The stopping and Range of lons in Solids, N. Y.: Pergamon, 1985.

[14] O. Debieu, R. P. Nalini, J. Cardin, X. Portier, J. Perrière and F. Gourbilleau, "Structural and optical characterization of pure Si-rich nitride thin films," Nanoscale Res. Lett., vol. 8, no. 31, p. 13, 2013.

[15] G. E. Jellison Jr. and F. A. Modine, "Parametrization of the optical functions of amorphous materials in the interband region," Appl. Phys. Lett., vol. 69, p. 371, 1996.

[16] “Erratum," Appl. Phys. Lett., vol. 69, p. 2137, 1996.

[17] M. C. Ridgway, T. Bierschenk, R. Giulian, B. Afra, M. D. Rodriguez, L. L. Araujo, A. P. Byrne, N. Kirby, O. H. Pakarinen, F. Djurabekova, K. Nordlund, M. Schleberger, O. Osmani, N. Medvedev, B. Rethfeld and P. Kluth, "Tracks and Voids in Amorphous Ge Induced by Swift Heavy-lon Irradiation," Phys. Rev. Lett., vol. 110, no. 24, p. 245502, 2013.

[18] F. Zhang, J. llavsky, G. G. Long, J. P. G. Quintana, A. J. Allen and P. R. Jemian, "Glassy carbon as an absolute intensity calibration standad for small-angle scattering," Metall. Mater. Trans., vol. 41, no. 5, pp. 1151-1158, 2009.

[19] P. Kluth, O. H. Pakarinen, F. Djurabekova, R. Giulian, M. C. Ridgway, A. P. Byrne and K. Nordlund, "Nanoscale density fluctuations in swift heavy ion irradiated amorphous SiO2," J. Appl. Phys., vol. 110, no. 12, p. 123520, 2011.

[20] B. Afra, K. Nordlund, M. D. Rodriguez, T. Bierschenk, C. Trautmann, S. Mudie and P. Kluth, "Thermal response of nanoscale cylindrical inclusions of amorphous silica embedded in alphaquartz," Phys. Rev. B, vol. 90, p. 224108, 2014.

[21] A. A. Leino, S. L. Daraszewicz, O. H. Pakarinen, K. Nordlund and F. Djurabekova, "Atomistic twotempreature modelling of ion track formation in silicon dioxide," EPL, vol. 110, no. 1, p. 16004, 2015.

[22] I. M. Lifshits, M. I. Kaganov and L. V. Tanatarov, "On the theory of radiation-induced changes in metals," J. Nucl. Energy. Part A. Reactor Science, vol. 12, no. 1, pp. 69-78, 1960.

[23] M. P. R. Waligorski, R. N. Hamm and R. Katz, "The radial distribution of dose around the path of beavy ion in liquid water," Radiat. Meas., vol. 11, no. 6, pp. 309-319, 1986.

[24] C. Dufour, V. Khomenkov, G. Rizza and M. Toulemonde, "Ion-matter interaction: the threedimensional version of the thermal spike model. Application to nanoparticle irradiation with swift heavy ions," J. Phys. D, vol. 45, no. 6, p. 065302, 2012.

[25] K. Awazu, X. Wang, M. Fujimaki, J. Tominaga, H. Aiba, Y. Ohki and T. Komatsubara, "Elongation of gold nanoparticles in silica glass by irradiation with swift heavy ions," Phys. Rev. B, vol. 78, no. 5, p. 054102, 2008.

[26] M. Toulemonde, C. Dufour, A. Meftah and E. Paumier, "Transient thermal processes in heavy ion irradiation of crystalline inorganic insulators," Nucl. Instr. Meth. Phys. Res. B, Vols. 166-167, pp. 903-912, 2000. 
[27] X. Zhang and C. P. Grigoropoulos, "Thermal conductivity and diffusivity of free-standing silicon nitride thin films," Rev. Sci. Instrum., vol. 66, no. 2, p. 1115, 1995.

[28] M. Bogner, A. Hofer, G. Benstetter, H. Gruber and R. Y. Fu, "Differential 3w method for measuring thermal conductivity of AIN and Si3N4 thin films," Thin Solid Films, Vols. 591, Part B, pp. 267-270, 2015.

[29] A. J. Griffin Jr. and F. R. Brotzen, "The effective transverse thermal conductivity of amorphous Si3N4 thin films," J. Appl. Phys., vol. 76, no. 7, p. 4007, 1994.

[30] Y. Ben-Hai and C. Dong, "Predictions of pressure-induced structural transition, mechanical and thermodynamic properties of alpha- and beta- Si3N4 ceramics: ab initio and quasi-harmonic Debye modeling," Chin. Phys. B, vol. 21, no. 6, p. 060508, 2012.

[31] F. de Brito Mota, J. F. Justo and A. Fazzio, "Structural properties of amorphous silicon nitride," Phys. Rev. B, vol. 58, no. 13, pp. 8323-8328, 1998.

[32] T. Watanabe , D. Yamasaki, K. Tatsumara and I. Ohdomari, "Improved interatomic potential for stressed Si, O mixed systems," Appl. Surf. Sci., vol. 234, no. 1-4, pp. 207-213, 2004.

[33] J. Samela, K. Nordlund, V. N. Popok and E. E. Campbell, "Origin of complex impact craters on native oxide coated silicon surfaces," Phys. Rev. B, vol. 77, no. 7, p. 075309, 2008.

[34] S. Munetoh, T. Motooka, K. Moriguchi and A. Shintani, "Interatomic potential for Si-O systems using Tersoff parameterization," Comput. Mater. Sci., vol. 39, no. 2, pp. 334-339, 2007.

[35] K. Nordlund, M. Ghaly, R. S. Averback, M. Caturla, T. Diaz de la Rubia and J. Tarus, "Defect production in collision cascades in elemental semiconductors and fcc metals," Phys. Rev. B, vol. 57, no. 13, pp. 7556-7570, 1998.

[36] H. J. C. Berendsen, J. P. M. Postma, W. F. van Gunsteren, A. DiNola and J. R. Haak, "Molecular dynamics with coupling to an external bath," J. Chem. Phys., vol. 81, no. 8, p. 3684, 1984.

[37] F. Djurabekova and K. Nordlund, "Atomistic simulation of the interface structure of $\mathrm{Si}$ nanocrystals embedded in amorphous silica," Phys. Rev. B, vol. 77, no. 11, p. 115325, 2008.

[38] I. Mariella and S. Meloni, "Atomistic structure of amorphous silicon nitride from classical molecular dynamics simulations," Phys. Rev. B, vol. 83, no. 16, p. 165209, 2011.

[39] G. Dupont, H. Caquineau, B. Despax, R. Berjoan and A. Dollet, "Structural properties of N-rich a-Si-N:H films with a low electron-trapping rate," J. Phys. D, vol. 30, pp. 1064-1076, 1997.

[40] K. C. Lin and S. C. Lee, "The structural and optical properties of a-SiNx:H prepared by plasmaenhanced chemical-vapor deposition," J. Appl. Phys., vol. 72, no. 11, pp. 5474-5482, 1992.

[41] A. Meftah, F. Brisard, J. M. Constantini, M. Hage-Ali, J. P. Stowuert, F. Studer and M. Toulemonde, "Swift heavy ions in magnetic insulators: A damage-cross-section velocity effect," Phys. Rev. B, vol. 48, no. 2, pp. 920-925, 1993.

[42] N. Tomozeiu, "Silicon Ixide (SiOx, $0<x<2$ ): a Challenging Material for Optoelectronics," in Optoelectronics-Materials and Techniques, InTech, 2011, pp. 55-100. 
[43] Z. G. Wang, C. Dufour, E. Paumier and M. Toulemonde , "The Se sensitivity of metals under swift-heavy-ion irradiation: a transient thermal process," J. Phys. D, vol. 6, pp. 6733-6750, 1994.

[44] G. Scardera, T. Puzzer, G. Conibeer and M. A. Green, "Fourier transform infrared spectroscopy of annealed silicon-rich silicon nitride thin films," J. Appl. Phys., vol. 104, no. 10, p. 104310, 2008.

[45] L.-N. He, D.-M. Wang and S. Hasegawa, "A study of plasma-deposited amorphous SiOx:H $(0<x<2)$ films using infrared spectroscopy," J. Non-Cryst. Solids, vol. 261, no. 1-3, pp. 67-71, 2000 .

[46] J. Fu, H. Shang, Z. Li, W. Wang and D. Chen, "Thermal annealing effect of the stress stability in silicon dioxide films grown by plasma-enhanced chemical vapor deposition," Microsys. Technol., vol. In Press, pp. 1-5, 2016.

[47] K. Awazu, S. Roorda, J. L. Brebner, S. Ishii and K. Shima, "Structure of latent tracks created by swift heavy ion in amorphous $\mathrm{SiO} 2$ and Zinc phosphate glass," Jpn. J. Appl. Phys., vol. 42, p. 3950, 2003.

[48] B. Canut, A. Ayari, K. Kaja, A.-L. Deman, M. Lemiti, A. Fave, A. Souifi and S. Ramos, "Ion-induced tracks in amorphous Si3N4 films," Nucl. Instr. Meth. Phys. Res. B, vol. 266, pp. 2819-2923, 2008.

[49] P. Mota-Santiago, F. Kremer, A. Nadzri, M. C. Ridgway and P. Kluth, "Elongation of metallic nanoparticles at the interface of silicon dioxide and silicon nitride," Nucl. Instr. Meth. Phys. Res. B, vol. In Press, 2017.

[50] J. E. Shelby, Introduction to Glass Science and Technology,, Second Edition ed., Cambridge: The Royal Society of Chemistry, 1997.

[51] A. Kushima, X. Lin, J. Li, X. Qian, J. Eapen, J. Mauro, P. Diep and S. Yip, "Computing the viscosity of supercooled liquids. II. Silica and strong-fragile crossover behaviour," J. Chem. Phys., vol. 131, p. 164505, 2009.

[52] Y. M. Haddad, Composites, Advanced Multilayered and Fibre-Reinforced, Netherlands: Springer, 2012.

[53] K. V. Loiko, I. V. Peidous, Y. Zu and H. M. Ho, "Experimental study of Si3N4 viscosity for calibration of stress-dependent models of silicon oxidation," J. Electrochem. Soc., vol. 146, no. 11, pp. 4226-4229, 1999.

[54] K. Awazu, S. Ishii, K. Shima, S. Roorda and J. L. Brebner, "Structure of latent tracks created by swift heavy-ion bombardment of amorphous SiO2," Phys. Rev. B, vol. 62, no. 6, pp. 3689-3698, 2000 .

[55] K. Awazu, S. Roorda, J. L. Brebner, S. Ishii and K. Shima, "Structure of latent tracks created by swift heavy ions in amorphous $\mathrm{SiO} 2$ and Zinc Phosphate glass," Jpn. J. Appl. Phys., vol. 42, p. 3950, 2003. 
[56] M. Toulemonde, W. J. Weber, G. Li, V. Shutthanandan, P. Kluth, T. Yang, Y. Wang and Y. Zhang, "Synergy of nuclear and electronic energy losses in ion-irradiation processes: The case of vitreous silicon dioxide," Phys. Rev. B, vol. 83, p. 054106, 2011.

[57] M. C. Busch, A. Slaoui, P. Siffert, M. Dooryhee and M. Toulemonde, "Structural and electrical damage induced by high-energy heavy ions in SiO2/Si structures," J. Appl. Phys., vol. 71, no. 6, p. 2596, 1992. 